\title{
Book Review \\ Two Degrees: The Built Environment and Our Changing Climate
}

McGregor, Alisdair, Roberts, Cole \& Cousins, Fiona, Abingdon: Routledge, 2013, ISBN 97800415-69300-4 (paperback, i - xviii, 1- 258 pages), USD 54.95 (hard cover 170.00, e-Book 54.95), GBP 34.99 (hard cover 105.00, e-Book 34.99)

As an academic and not an architectural practitioner, and as a theorist but with no connections to issues of climate change, energy efficiency, sustainability, greenhouse gas emissions, "green buildings', etc., this book offers an interesting and informative introduction to just such issues. Whether those already expert in the field - or perhaps those opposing such issues, for anticlimate change or for purely formalistic reasons - might have quite different views on the quality of the book is, of course, a different matter.

Interestingly, Cole Roberts starts his Preface with a dual contention: first, that "There are many books on theory. This is not one" [xv]; and second, that the purpose of this book is about "...stepping into the practice of creating buildings, infrastructure, and communities that will last us into a human-stewarded future" [xv]. For a non-expert, albeit one who has written about the importance of social benefit in relation to architecture and urban design, both are of significance. While theory is certainly of importance in its own right, it is, as Roberts suggests, of no real value in a book in presenting its readership with positive and pragmatic and information-based data that allows for a more well-developed and potentially effective understanding of actually how to address such issues.

Divided into three sections, Fundamentals, Mitigation Strategies, and Adaptation Strategies, the book offers 20 chapters that take the reader from an introduction to 'The Science of Climate Change' to a series of guidelines on issues affecting design for different climates and communities. For the non-expert, Part 1 on Fundamentals offers perhaps the most significant information, although for those with extant knowledge and a desire to explore notions of 'how to' from a more effective and more sophisticated perspective, Part 2 on Mitigation Strategies offers greater benefit. A degree of selectivity is likely to be directed at Part 3 on Adaptation Strategies based on the kind(s) of climate one wishes to explore and the information one needs to address particular issues, e.g. 'Designing for Warmer and Wetter Climates' (Chapter 17) versus 'Designing for Hotter and Drier Climates' (Chapter 18).

Focussing, then, on the five chapters within Fundamentals, the material provided is both informative and essentially easy to understand. This is not to suggest that the data is in any way simplistic; rather that the extent of the 'new' reader's understanding is in some degree linked to their existing background knowledge. As a potential information source, indeed as a possible textbook, for university students and/or professionals without an existing and comprehensive knowledge of the effects and significance of climate change, and our ways of dealing with it, the opening section of the book provides a comprehensive and well-explained set of information about just such issues. At the same time - and relating to the book as a whole, as well as a number of others - what is also significant is how, in relation to climate change, notions of position and opinion, preference and prejudice, might cause readers to accept and/or reject the information provided.

Nevertheless, from the perspective of one who is concerned about the issues of climate change, and, perhaps more importantly, seeks direct and comprehensible information and explanations 
about its nature and how such issues can be addressed, then Two Degrees is in many ways both an excellent starting point for those seeking 'basic' understanding, and an informative introduction to the pragmatics of climate change strategies. Chapter 1, 'The Science of Climate Change' (pp.2-17), thus provides the reader with a short, sharp and highly focussed set of information about global warming and the rise in the earth's temperature, based on clear writing and augmented with useful graphs and diagrams. The notion of position mentioned above, and thus the idea of scepticism, indeed potential opposition and outright rejection of climate change 'ideas', is usefully discussed in Hacker \& Roberts' brief section on 'How Certain is the Science?' (pp.9-10). While some readers may not accept the issues raised, they surely must attempt to engage with and dispute these issues, rather than simply rejecting them! The subsequent section, 'Part 2 of the Global Warming Hypothesis' (pp.10-14) then offers a clear and concise analysis of what global warming is, and what its impacts are likely to be.

As the title suggests - and again perhaps for the non-expert - Chapter 2, Cousins' 'Greenhouse Gas Emissions in the Built Environment' (pp.18-25), clearly explains the issues of what greenhouse gas emissions are, their extent, and provides detailed information on a range of such emissions. Significantly, albeit very briefly, the inclusion of the 'Case Study: One Building, Many Choices - Total Emissions Footprint' offers a useful introduction to the effects on the built environment "...to help visualize the relative importance of transportation, operational and embodied energy use" (p.22). Essentially simply a table, the data provided is such that it ought not only to attract building designers, architects, planners, developers, councils, etcetera, but also cause them to seriously consider the issues raised.

While it must be admitted that the chapters are extremely short, this is not a bad thing. The book is coherent and well-structured, and provides useful information in a very direct and easily understandable manner. Moreover, the chapters 'move' from scientific information on climate change, to issues associated with the built environment, and thus to Chapter 3 which deals with 'Policies to Mitigate Climate Change' (pp.26-39). This now begins to offer the reader a more direct focus on international policy in relation to climate change, such as the Kyoto Protocol, and the problems arising from its 'follow-up', and a very useful set of ideas about 'Major Policy Mechanisms' (pp.30-35). Perhaps even more useful is information on a range of 'Specific Policies affecting the Built Environment' (pp.35-38) - the UK, US and China. While these are again very brief, the idea of presenting them not only gives the reader an understanding of the differences between them and what they might 'do', but, perhaps more importantly, allows the reader to search for more detailed information in a range of other sources.

Chapters 4 ('Sustainability and Climate Change', pp.40-47) and 5 ('Mitigation and Adaptation', pp.48-53) extend the information provided on how the reader - and, again, we might say here the potentially 'novice' reader - might come to understand climate change, sustainable design, resource management, and the serious issue of potential strategies. The latter are covered in more detail, although still in short but focussed chapters, in Part 2 on Mitigation Strategies. This takes the reader beyond mere 'basic' information and begins to establish what it is we might or should - do in relation to solving issues of climate change, sustainable environments, and zero-carbon buildings, as well as considering problems - and solutions - associated with communities in general, with ameliorating carbon emissions from existing building stock, with the notion of integrated design, and with evaluating strategies and making considered choices.

Two Degrees is thus to be highly recommended. While it may comprise a selection of short chapters, or may be considered 'thin' by a variety of experts who might expect more, the book offers an important range of information concerning climate change and strategies for addressing such issues; does so in a way that may be brief but is well-written, highly-focussed 
and easily understood; offers the potentially 'new' reader a degree of comprehension concerning significant issues without being too extensive and too complex; and allows for an expansion of such understanding by suggesting reference to additional books that are more detailed in relation to very specific issues. All-in-all, an intriguing and informative book, and, potentially, a very useful 'textbook' for student and professional alike.

Associate Professor Steve Harfield

University of Technology, Sydney 